a Universidade Federal do Paraná, Departamento de Engenharias e Exatas, Setor Palotina, CEP 85950-000, PalotinaPR, Brasil.

*E-mail: fernandabbach@ gmail.com

Recebido: 2 de Abril de 2021

Aceito: 27 de Julho de 2021

Publicado online: 28 de Julho de 2021

\section{A Influência de Políticas Públicas para o Progresso da Geração Solar Fotovoltaica e Diversificação da Matriz Energética Brasileira}

\author{
The Influence of Public Policies for the Progress of Photovoltaic Solar \\ Generation and Diversification of The Brazilian Energy Matrix
}

\author{
Fernanda Bach Gasparin, ${ }^{a} *$ (1) Victória Oliveira Diaz de Lima, ${ }^{a}$ Débora Hungaro Micheletti, ${ }^{a}$
} Eduardo Lucas Konrad Burin ${ }^{2}$

In the current Brazilian scenario, it is possible to see clearly the importance of public policies for the promotion of renewable energies. Nevertheless, the existing policies are not yet effective for clean energy, including solar photovoltaic, to reach its potential. In general, the stimulus of photovoltaic solar energy production has great advantages because in Brazil there is an extensive territory with large irradiation levels and a high potential for electricity generation. In addition, the diversification of the electric matrix is essential for good management of this sector, guaranteeing the supply of energy to meet the demand, safety, and energy efficiency. As inspiration can be cited China and Germany, countries that have made great advances in the energy matrix in recent years due to their public policies to encourage the generation of renewable energy. Moreover, photovoltaic solar energy generates the highest number of jobs per megawatt, being essential for the country's economic development. Through bibliographic research, the present work sought to evaluate the existing public policies in Brazil, as well as in China and Germany, in order to understand their impacts on the development of photovoltaic solar energy. Thus, it was realized the need for better government planning so that the potential of Brazilian solar generation is reached.

Keywords: Public policies; renewable energy; solar photovoltaic energy.

\section{Introdução}

Atualmente o uso de energia no mundo é baseado principalmente em fontes não renováveis, como petróleo, gás natural e carvão. Esses combustíveis, além de esgotáveis, são fontes de geração de gases do efeito estufa e colocam em risco a sustentabilidade do planeta. ${ }^{1}$

Nesse contexto, a preocupação ambiental juntamente com o desenvolvimento sustentável tornou-se foco após a vigência do Protocolo de Kyoto, o qual estabelece que os países desenvolvidos devem reduzir a emissão de dióxido de carbono através do aumento da eficiência energética, além da pesquisa, promoção, desenvolvimento e uso de energias renováveis. Para países em desenvolvimento, a utilização de energias renováveis significa um importante instrumento de crescimento econômico. ${ }^{2}$

Além disso, a diversificação da matriz elétrica tem sido estimulada mundialmente com o auxílio de políticas públicas, adotadas por diferentes países com o objetivo de obter confiabilidade e disponibilidade no sistema elétrico, bem como atender às diretrizes do desenvolvimento sustentável. ${ }^{3}$

No Brasil, apesar da grande contribuição das energias renováveis, é possível notar que não há uma grande diversificação da matriz elétrica, o que pode ser explicado pelo baixo custo da energia gerada pelas usinas hidrelétricas, a principal fonte elétrica do país, quando comparada a outras fontes de energia. ${ }^{3}$

Esse cenário poderia ser transformado com o uso de políticas públicas que buscassem o desenvolvimento na produção e distribuição de tecnologia, promovendo a redução dos custos de implantação de outras fontes de energia elétrica. Nesse sentido, é possível observar os inúmeros recursos ambientais que o Brasil possui e que possibilitariam a diversificação do setor elétrico de forma estratégica. Dentre esses recursos, a energia solar fotovoltaica ganha destaque, não só pelos altos índices de irradiação observados em boa parte do território nacional, mas também por possuir viabilidade de implantação em pequena escala, por meio da geração distribuída instaladas nas próprias unidades de consumo, quando comparada a outras fontes de energia. ${ }^{2}$

Esse fato, agregado com políticas públicas para garantir mercado e tornar essa tecnologia acessível financeiramente, pode impulsionar fortemente a sua implementação e proporcionar a diversificação da matriz elétrica brasileira. ${ }^{2}$ 
Dessa forma, o objetivo desse estudo foi avaliar o impacto das políticas públicas relacionadas à energia solar fotovoltaica existentes no mundo, especialmente no Brasil, a fim de compreender sua importância para o progresso dessa fonte de energia, bem como para a diversificação da matriz elétrica nacional.

\section{Metodologia}

O presente estudo foi realizado com base em uma pesquisa qualitativa, através do uso de material bibliográfico, como artigos, monografias e dissertações, obtidas por meio da pesquisa de palavras chaves, como energia solar e políticas públicas, sendo analisados documentos que tratam de assuntos referentes a políticas públicas de incentivo a energia solar fotovoltaica tanto no Brasil quanto no exterior, bem como gestão energética e geração de empregos. Além disso, a pesquisa baseou-se em consultas nas Resoluções Normativas da ANEEL, bem como dados da Renewable Energy Policy Network for the 21st Century (REN21) e da Agência Internacional de Energias Renováveis (IRENA) sobre a participação da energia solar fotovoltaica na matriz elétrica brasileira e mundial.

\section{A Energia Solar Fotovoltaica}

A energia solar fotovoltaica é obtida através do efeito fotovoltaico, que consiste na conversão direta da luz solar em energia elétrica por meio de células fotovoltaicas, sendo considerada uma alternativa promissora para expansão da oferta de energia elétrica com baixo impacto ambiental. Além disso, essa fonte de energia permite instalações em pequena escala e em áreas remotas, não necessitando de investimento em linhas de transmissão, além de também poder ser conectada com a rede. ${ }^{4}$

Além desses benefícios existem outros: a redução dos gastos com energia elétrica; geração de empregos locais de qualidade; geração de energia limpa, renovável e sustentável; contribuição para atingir as metas de redução de emissão de $\mathrm{CO}_{2}$; diversificação da matriz elétrica; ampliação do uso de energias renováveis no país; redução de perdas por transmissão e distribuição de energia elétrica. ${ }^{5}$

Uma das vantagens da energia solar fotovoltaica está diretamente relacionada ao fato de que essa energia pode ser enquadrada tanto como geração centralizada quanto como geração distribuída. Nesse sentido, a geração centralizada é considerada a mais tradicional, baseando-se no emprego de grandes fontes geradoras para produzir energia elétrica, chegando ao consumidor por meio de linhas de transmissão e distribuição. Já a geração distribuída é baseada em uma fonte de geração de energia elétrica junto ou próximo ao próprio consumidor, onde o excedente é encaminhado à rede de transmissão. ${ }^{6}$

Outro fator importante é que em 2015, segundo dados da IRENA, houve 8,1 milhões vagas de emprego geradas mundialmente relacionadas a energia renovável ${ }^{7}$, enquanto em 2016 esse valor subiu para 9,8 milhões de empregos, dentre os quais 3,95 milhões dessas vagas estão relacionadas a energia solar fotovoltaica. ${ }^{2}$ Vale ressaltar que para cada megawatt instalado de geração fotovoltaica, são necessários de 25 a 30 novos empregos, distribuídos entre instaladores, projetistas, fabricantes e montadores do sistema. ${ }^{7}$

\subsection{Panorama atual de geração de energia fotovoltaica no Brasil}

A energia solar é a que mais cresce na matriz energética mundial. Atualmente, a capacidade mundial instalada de energia solar fotovoltaica é de 580,16 GW. Os países com as maiores capacidades instaladas de energia solar fotovoltaica em 2019 eram China (205,07 GW), Estados Unidos (60,54 GW), Japão $\left(61,84\right.$ GW), Alemanha (48,96 GW) e Índia $\left(34,8\right.$ GW) ${ }^{8}$

Já o Brasil, até dezembro de 2020, possuía 2,989 GW de potência instalada em geração centralizada e 4,254 GW de potência instalada em geração distribuída em energia solar fotovoltaica. ${ }^{9}$

Nesse contexto, o crescimento acentuado da capacidade instalada da geração solar fotovoltaica tem promovido uma maior diversificação da matriz elétrica brasileira, garantindo maior segurança, qualidade e confiança no sistema elétrico nacional, além de representar um ponto estratégico para o desenvolvimento econômico do país. ${ }^{2}$

É necessário reconhecer, ainda, que as condições climáticas do Brasil são fortemente favoráveis para o aumento da participação da energia solar fotovoltaica na diversificação das formas de obtenção de energia elétrica. ${ }^{7}$

De forma geral, no Brasil há uma elevada incidência de radiação solar em praticamente todo o território e, apesar de ainda ser considerada uma solução cara frente a outras formas de energia, a geração solar fotovoltaica é a que apresenta maior taxa de crescimento e queda de custos. ${ }^{10} \mathrm{Em} 2010$, os custos dos módulos fotovoltaicos eram $90 \%$ superiores comparado a 2018 , enquanto a capacidade mundial de geração fotovoltaica cresceu de $40 \mathrm{GW}$ em 2010 para 505 GW em 2018. ${ }^{11}$

Quando se analisa a irradiação solar global incidente no país, observa-se que a maior incidência ocorre no norte do estado da Bahia, atingindo até $6,50 \mathrm{kWh} / \mathrm{m}^{2}$ por dia. Por outro lado, o local com menor incidência se dá no litoral do estado de Santa Catarina, com valor de $4,25 \mathrm{kWh} / \mathrm{m}^{2}$ por dia. ${ }^{4}$ Importante notar, entretanto, que a incidência solar no litoral de Santa Catarina representa cerca de $40 \%$ mais radiação do que os melhores índices encontrados na Alemanha, país referência em implementação e uso dessa energia, o que demonstra o grande potencial de geração presente no Brasil. ${ }^{2}$

\section{Políticas Públicas}

As políticas públicas de incentivo à utilização de fontes de energia renovável e de eficiência energética são fatores chave para viabilizar o desenvolvimento sustentável. ${ }^{12}$ 
Além disso, direcioná-las ao aperfeiçoamento da produção e distribuição de tecnologia estimula o uso de fontes renováveis de energia e reduz seus custos. ${ }^{2}$

O mercado de energia elétrica pode sofrer influência do Estado através da criação de políticas energéticas, da definição do planejamento energético bem como da normatização de mercados de energia. De forma geral, as políticas públicas demonstram quais as prioridades do governo para que o setor energético se desenvolva. ${ }^{13}$

Os principais mecanismos de incentivo à utilização de energias renováveis utilizados pelos governos estão descritos na Tabela 1.

\subsection{Políticas públicas no Brasil}

Em relação a políticas públicas que incluem a energia solar fotovoltaica, tem-se o primeiro marco em $2012 \mathrm{com}$ a implementação das Resoluções Normativas (REN) $n^{\circ} 481$ e 482 da Agência Nacional de Energia Elétrica (ANEEL).

Respectivamente, a primeira norma estabeleceu que projetos com potência injetada na rede menor ou igual a 30 MW tenham descontos de $80 \%$ nas Tarifas de Uso de Transmissão e Tarifas de Uso de Distribuição nos 10 primeiros anos de operação, desde que entrassem em operação até o final de 2017. A partir do início de 2018 o desconto seria de 50\%. ${ }^{16}$

Já a segunda norma buscou estimular a geração distribuída de energia elétrica através de micro e mini geradores para autoconsumo. Além disso, ela também estabelece os princípios gerais de créditos energéticos, compensação energética (net metering), encargos, potência e normas relacionadas à micro e minigeração distribuída. Essa REN foi reformulada e alterada para a Resolução Normativa $\mathrm{n}^{\mathrm{o}}$ 687/2015. ${ }^{17}$

Outro incentivo foi dado pelo Conselho Nacional de Política Fazendária (CONFAZ) através dos Convênios $n^{\circ}$ $101 / 1997$ e $n^{\circ} 16 / 2015$ que isenta o ICMS para alguns equipamentos utilizados para geração de energia elétrica por fonte solar ou eólica e isenta os estados de cobrar ICMS sobre a energia injetada na rede. ${ }^{6}$ Atualmente todos os estados aderiram à isenção dada pelo Convênio no ${ }^{\circ}$ 16/2015.

Em relação à Geração Centralizada, o país buscou incluir a energia solar fotovoltaica em leilões de energia. A primeira inclusão ocorreu no $6^{\circ}$ Leilão de Energia de Reserva (LER/2014), tendo participação garantida desde então. ${ }^{18}$
Outro meio de incentivo para a geração distribuída foi o estabelecimento de condições de financiamento diferenciadas para a aquisição de sistemas fotovoltaicos. Nesse sentido, em 2015 o Ministério de Minas e Energia (MME) lançou o Programa de Desenvolvimento da Geração Distribuída de Energia Elétrica - ProGD com o objetivo de promover a ampliação da geração distribuída com base em fontes renováveis. Com isso, alguns bancos públicos e instituições privadas lançaram linhas de crédito para financiamento na aquisição de sistemas fotovoltaicos, como o Banco Nacional de Desenvolvimento Econômico e Social (BNDES), Banco da Amazônia, Banco do Brasil, Caixa Econômica Federal, Bradesco, Santander, BV Financeira, Sicoob, entre outros. ${ }^{6}$

Embora exista um número considerável de incentivos para a geração solar fotovoltaica e um grande crescimento nesse setor nos últimos anos, ainda há muitos incentivos necessários para que a energia solar se consolide na matriz elétrica nacional..$^{15} \mathrm{O}$ que encarece os empreendimentos de geração renovável é o investimento inicial, fazendo-se necessárias políticas de crédito e incentivos fiscais mais eficientes. ${ }^{2}$

Bons mecanismos de compensação de energia gerada em sistemas distribuídos são imprescindíveis para que ocorra a efetiva ampliação da fonte solar fotovoltaica. Os mesmos autores expressam a necessidade de realizar uma análise ampla e estruturada dos custos existentes da implementação de sistemas de compensação e de incentivos à geração fotovoltaica, evitando assim problemáticas econômicas e sociais devido a alocação de benefícios, tendo em vista que o reequilíbrio financeiro das distribuidoras de energia elétrica ocorreria com a elevação das tarifas. ${ }^{19}$

\subsection{Políticas públicas no exterior}

Como exemplos de políticas públicas eficientes, pode-se citar o caso da China, país líder em capacidade instalada de energia solar fotovoltaica em 2018, ${ }^{11}$ e Alemanha, cuja energia fotovoltaica tem considerável participação na matriz elétrica do país.

\subsection{China}

A China buscou alternativas para produção de energia renovável pois sofria uma forte pressão da comunidade

Tabela 1. Políticas públicas de incentivo a geração de energias renováveis tipicamente empregadas no mundo

\begin{tabular}{ll}
\hline Mecanismo & Funcionamento \\
\hline Sistema feed in & $\begin{array}{l}\text { O produtor de energia elétrica renovável é remunerado pelas empresas de energia recebendo o valor de tarifas especiais que o } \\
\text { governo estipulou, que são garantidas por um determinado período. }\end{array}$ \\
Sistema de leilão & $\begin{array}{l}\text { O governo determina a quantia de energia renovável a ser leiloada e produzida, sendo um sistema competitivo na qual os } \\
\text { vencedores são os que apresentam propostas com menor valor, com esse valor garantido por um longo prazo. }{ }^{14}\end{array}$ \\
Sistema de cotas & $\begin{array}{l}\text { O governo estipula uma quantidade de energia renovável que as empresas de energia devem fornecer, podendo produzir } \\
\text { diretamente essa energia ou comprando certificados verdes. }{ }^{14}\end{array}$ \\
Sistema net metering & $\begin{array}{l}\text { O usuário utiliza a energia renovável produzida para compensar parcialmente ou todo seu consumo de energia da rede } \\
\text { elétrica. Esse sistema é utilizado no Brasil desde } 2012 .{ }^{15}\end{array}$ \\
\hline
\end{tabular}

Fonte: O autor, 2021 
internacional para que a emissão de $\mathrm{CO}_{2}$ fosse reduzida. A proposta era de que até 2020 a redução dióxido de carbono seria de $45 \%$ por unidade de PIB em relação a $2005 .{ }^{20}$

O país, que atualmente conta com a maior produção de energia solar no mundo, teve uma importante política pública iniciada em 2006 com a Lei de Energias Renováveis, a qual visou o desenvolvimento da energia eólica e solar utilizando o mecanismo de tarifa feed in. Essa lei foi revisada em 2009 para garantir a compra de energia elétrica renovável pelas companhias de energia. ${ }^{6}$

No mesmo ano, o governo criou os programas Golden Sun e Building Integrated PhotoVoltaics (BIPV), além de projetos de larga escala dentro da tarifa feed in. O BIPV buscou subsidiar os custos de implantação dos sistemas fotovoltaicos em edifícios e fachadas. Já o programa Golden Sun foi responsável pelo estímulo à indústria fotovoltaica, pois estabelecia subsídios fiscais, apoio científico e tecnológico. ${ }^{20}$ Nesse programa, o subsídio de energia solar era de $50 \%$ em projetos ligados à rede e de $70 \%$ em regiões remotas, além de empréstimos a juros reduzidos e redução de impostos. ${ }^{6}$

Com essas políticas públicas houve um crescimento no mercado interno e uma redução drástica nos valores dos produtos fotovoltaicos, o que refletiu no aumento de investidores estrangeiros, possibilitando uma expansão contínua nesse setor. Em 2015, a China proporcionou uma reforma no mercado de energia, passando a dar prioridade ao uso de energia renovável e buscando aumentar o desenvolvimento desse setor em províncias com maior demanda de energia. ${ }^{20}$

Depois de alguns ajustes da tarifa feed in, o país chegou ao final de 2017 com 130,80 GW instalados. Em 2018, adicionou mais $45 \mathrm{GW}$ e ao final de 2019, essa capacidade totalizou 205,07 GW, mantendo-se na posição de país com maior desenvolvimento fotovoltaico. ${ }^{8}$

\subsection{Alemanha}

A Alemanha é considerada pioneira na busca de políticas públicas eficientes voltadas especificamente para energias renováveis. Esse crescimento se deve, em grande parte, pela adoção das tarifas feed in. ${ }^{2}$

Essas ações tiveram início em 1991, com a vigência da Lei de Alimentação de Energia, na qual o governo estabeleceu a compensação de energia renovável pelo mecanismo feed in. Nesse momento, a energia eólica se destacou devido ao seu menor custo, no entanto, o governo alemão criou o Programa 1.000 telhados com objetivo de garantir de 60 a $79 \%$ do financiamento de instalações de sistemas fotovoltaicos. Como o mercado ainda estava receoso, criou-se o Programa 100.000 Telhados que subsidia empréstimos a juros baixos para os investidores. ${ }^{6}$

O próximo grande passo foi dado com a implantação da Lei de Fontes Renováveis de Energia (EEG) de 2000, na qual foi feita uma revisão da tarifa feed in e determinou-se que a compra da energia renovável fosse distribuída entre as concessionárias a fim de evitar que algumas regiões fossem sobrecarregadas. Também foi definido que o valor da compra de cada $\mathrm{kWh}$ sería específico para diferentes fontes de energia, assegurando essa tarifa pelo período de 20 anos. $^{6}$

Essa Lei passou por algumas mudanças ao longo dos anos, sendo as mais significativas em 2004, 2009, 2012 e 2014, a fim de tornar o modelo feed in mais eficiente. As modificações se referiram às tarifas, obrigações das concessionárias de energia e metas de uso de energia renovável no país ao longo dos próximos anos. Já em 2017, ocorreu uma modificação mais brusca, na qual o governo passou a introduzir um sistema de leilões, onde as tarifas feed in teriam seus valores determinados. ${ }^{21}$

Pode-se considerar outros incentivos dados à energia solar fotovoltaica pelo governo alemão, como subsídios e incentivos financeiros, apoio para investimento em fábricas nacionais e empréstimos a juros reduzidos. Esse conjunto de ações possibilitou uma grande redução no preço de sistemas fotovoltaicos, no entanto o apoio governamental ainda é necessário para novos investimentos. ${ }^{6}$

As políticas públicas da Alemanha fizeram com que o país ganhasse destaque mundial e assumisse a posição de país com maior capacidade de energia solar fotovoltaica per capita, além de estar entre os cinco países com maiores produções de energia renovável no mundo. ${ }^{22}$

\section{Considerações Finais}

A energia solar fotovoltaica apresenta capacidade para ser uma aliada na diversificação da matriz elétrica brasileira, tendo em vista o grande potencial de irradiação solar em todo território nacional, muito acima da média dos países europeus. No entanto, apesar do crescimento de implementação de sistemas fotovoltaicos após a REN no 482/2012, ainda há problemas passíveis de melhorias. As políticas públicas brasileiras não são suficientes para o fomento dessa fonte de energia, tendo em vista o alto custo de investimento.

A análise de políticas públicas internacionais serve como uma forma de aprendizado para a implementação de políticas públicas nacionais eficazes. Tais políticas demonstram um forte apoio a pesquisa e desenvolvimento desse setor, bem como incentivos com subsídios e tarifas atrativas. Essas ações tornam o setor de energia fotovoltaica atrativo para investidores, garantindo a eles retorno financeiro vantajoso frente a outras possibilidades de investimento.

Sendo assim, é papel do governo intervir com ações efetivas a fim de tornar a energia solar fotovoltaica mais acessível para que ocorra uma maior diversificação da matriz elétrica nacional e, consequentemente, um maior desenvolvimento econômico para o país.

\section{Referências Bibliográficas}

1. Internacional Energy Agency, World Energy Balances: Overview, 2019. Disponível em: <https://www.iea.org/reports/ world-energy-balances-overview>. Acesso em: 20 dezembro 2020. 
2. Stefanello, C.; Marangoni, F.; Zeferino, C. L.; Resumos do VII Congresso Brasileiro de Energia Solar, Gramado, Brasil, 2018. [Link]

3. Tiepolo, G. M.; Junior, J. U.; Pereira, E. B.; Pereira, S. V.; Alves, A. R.; Resumos do Congresso Brasileiro de Planejamento Energético, Gramado, Brasil, 2016. [Link]

4. Silva, R. G.; Carmo, M. J. D.; Energia Solar Fotovoltaica: Uma proposta para melhoria da gestão energética. InterSciencePlace 2017, 12, 129. [Link]

5. Sítio da Associação Brasileira de Energia Solar Fotovoltaica no Brasil. Disponível em: <https://www.absolar.org.br/deixeasolarcrescer/wpcontent/uploads/2019/12/ohvQK.pdf $>$. Acesso em: 20 setembro 2020.

6. Pereira, R. C.; Trabalho de Conclusão de Curso, Instituto Federal de Educação, Ciência e Tecnologia de Goiás, 2019. [Link]

7. Santos, E. P.; Conti, T. N.; Mercado profissional para a área de energia e eficiência energética no Brasil. Revista Internacional de Ciências 2017, 7, 142. [Link]

8. Internacional Renewable Energy Agency, Renewable Capacity Statistics 2020. Disponível em: <https://irena.org/ publications/2020/Mar/Renewable-Capacity-Statistics-2020>. Acesso em: 16 dezembro 2020.

9. Sítio da Associação Brasileira de Energia Solar Fotovoltaica no Brasil. Disponível em: <https://www.absolar.org.br/mercado/ infografico/>. Acesso em: 20 setembro 2020.

10. Internacional Renewable Energy Agency, Renewable Power Generation Costs in 2019. 2020. Disponível em: <https://www. irena.org/publications/2020/Jun/Renewable-Power-Costsin-2019>. Acesso em: 21 dezembro 2020.

11. Ren21, Renewables 2019: Global Status Report. Disponível em: <https://www.ren21.net/wp-content/uploads/2019/05/ gsr 2019 full report en.pdf $>$. Acesso em: 21 dezembro 2020.
12. Lopes, M. C.; Taques, F. H.; O desafio da energia sustentável no Brasil. Revista Cadernos de Economia 2016, 20, 71. [Link]

13. Bajay, S. V.; Badanhan, L. F.; Conferência sobre Sustentabilidade na Geração e Uso de Energia no Brasil: os Próximos Vinte Anos, Campinas, Brasil, 2002. [Link]

14. Martins, J. M. C.; Dissertação de Mestrado, Universidade Estadual de Campinas, 2010. [Link]

15. Nascimento, R. L.; Energia Solar no Brasil: Situações e Perspectivas. Câmara dos Deputados, Consultoria Legislativa, 2017. [Link]

16. Sítio da Agência Nacional de Energia Elétrica. Disponível em: $<$ http://www2.aneel.gov.br/cedoc/ren2012481.pdf $>$. Acesso em: 13 dezembro 2020

17. Sítio da Agência Nacional de Energia Elétrica. Disponível em: $<$ http://www.aneel.gov.br/cedoc/ren2012481.pdf $>$. Acesso em: 13 dezembro 2020.

18. Sítio da Empresa de Pesquisa Energética. Disponível em: $<\underline{\text { https:// }}$ www.epe.gov.br/pt/leiloes-de-energia/leiloes/leilao-de-energiade-reserva-2014>. Acesso em: 13 dezembro 2020.

19. Castro, N. J.; Dantas, G.; Experiências Internacionais em Geração Distribuída: Motivação, Impactos e Ajustes, 1a. ed., Publit: Rio de Janeiro, 2018. [Link]

20. Cavalcante, R. C.; Dissertação de Mestrado, Universidade Federal da Paraíba, 2018. [Link]

21. Castro, L. M.; Monografia, Universidade Federal do Rio de Janeiro, 2018. [Link]

22. Ren21, Renewables 2017: Global Status Report. Disponível em: <https://www.ren21.net/wp-content/uploads/2019/05/ GSR2017_Full-Report_English.pdf>. Acesso em: 23 dezembro 2020. 